\title{
Impact of the carer on length of hospital stay for mental health: Results from two Australian surveys
}

Short title: Carers and length of hospital stay

Original article intended for: International Journal Mental Health Nursing

Authors:

Emily Hielscher

Sandra Diminic

Meredith Harris ${ }^{1,2}$

David Castle

Yong Yi Lee ${ }^{1,2}$

Jan Kealton ${ }^{5}$

Harvey Whiteford ${ }^{1,2}$

Affiliations:

1. Policy and Epidemiology Group, Queensland Centre for Mental Health Research (QCMHR), The Park

Centre for Mental Health, Brisbane, QLD, Australia

2. School of Public Health, Faculty of Medicine, The University of Queensland, Brisbane, QLD, Australia

3. Centre for Clinical Research, Faculty of Medicine, The University of Queensland, Brisbane, QLD, Australia

4. University of Melbourne and St Vincent's Hospital, Melbourne, VIC, Australia

5. Carer consultant

6. Institute of Health Metrics and Evaluation, University of Washington, Seattle, USA

Address for correspondence:

Emily Hielscher

The University of Queensland

UQ Centre for Clinical Research,

Building 71/918 RBWH Herston,

Brisbane QLD 4029, Australia

Email: e.hielscher@uq.edu.au; Facsimile: 073346 5509; Telephone: 0733465555

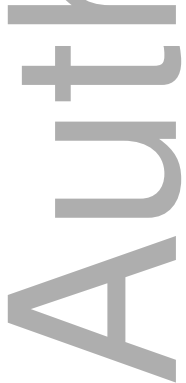

\section{Acknowledgements}

This is the author manuscript accepted for publication and has undergone full peer review but has not been through the copyediting, typesetting, pagination and proofreading process, which may lead to differences between this version and the Version of Record. Please cite this article as doi: $10.1111 /$ inm.12543

This article is protected by copyright. All rights reserved 
The authors thank members of the reference group established by Mind who provided advice and guidance on early versions of this work: Frances Sanders, Margaret Grigg, Philip Norman, and Gerry Naughtin.

The authors acknowledge the contribution of the mental health carers who participated in the UQ Carer Survey 2016 and the carer organisations who facilitated the recruitment of participants.

Parts of the results reported in this article are based on data collected in the framework of the 2010 Australian National Survey of High Impact Psychosis. The members of the Survey of High Impact Psychosis Study Group are: V. Morgan (National Project Director), A. Jablensky (Chief Scientific Advisor), A. Waterreus (National Project Coordinator), R. Bush, V. Carr, D. Castle, M. Cohen, C. Galletly, C. Harvey, B. Hocking, A.

Mackinnon, P. McGorry, J. McGrath, A. Neil, S. Saw, H. Stain. Ethics approvals for the study were obtained from relevant institutional human research ethics committees. The study was funded by the Australian Government Department of Health and Ageing. The authors acknowledge, with thanks, the hundreds of mental health professionals who participated in the preparation and conduct of the survey and the many Australians with psychotic disorders who gave their time and whose responses form the basis of this analysis.

\section{Funding}

This research was funded by Mind under the Mind Carer Development Fund as part of a broader project to estimate the replacement cost of mental health caring.

\section{Authorship statement}

$\mathrm{EH}, \mathrm{SD}$, and $\mathrm{MH}$ contributed to the conception and design of the paper. EH collected the data for the UQ Carer Survey, and analysed both datasets included in the manuscript (UQ Carer Survey and SHIP survey). EH and SD wrote the first draft of the manuscript. MH commented on the first manuscript and all authors contributed to the writing of subsequent versions. All authors contributed to and have approved the final manuscript.

\section{Declaration of Conflicting Interests}

The authors declare that there are no conflicts of interest.

Word count: 4996; tables: 3; references: 45

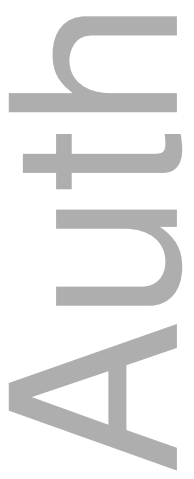


MS. EMILY HIELSCHER (Orcid ID : 0000-0002-0559-5256)
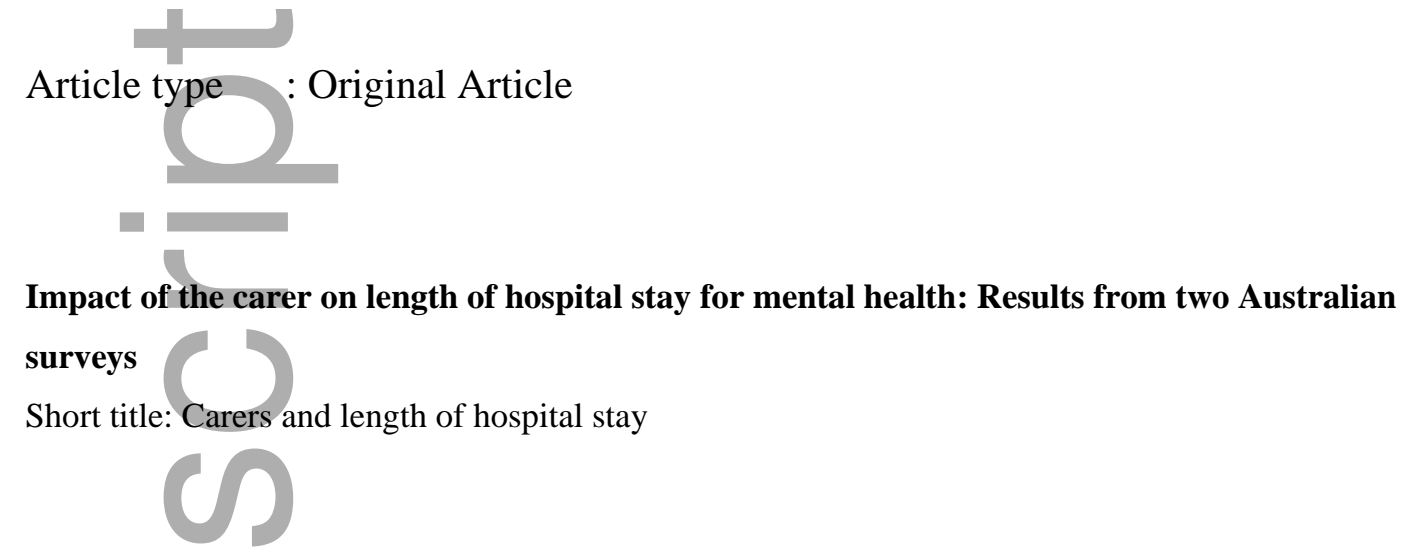

Abstract

Informal carers play a vital role in supporting Australians living with a mental illness, including during the acute phases of illness; however, little is known about their impact on length of hospital stay. We aimed to investigate the impact of having a carer and of carer burden on length of hospital stay for mental health. Two Australian datasets were used. Data from the 2010 National Survey of High Impact Psychosis $(n=1,825)$ were used to investigate the impact of having versus not having a carer on length of hospital stay for mental health. Data from the UQ Carer Survey 2016 ( $n=105)$, a convenience sample of mental health carers, were used to investigate the impact of weekly hours of care (a measure of objective carer burden) on length of stay. Multiple logistic regression and correlation analyses were performed to investigate the association between carer status/burden and length of stay. Having a carer was associated with a significantly longer length of hospital stay; however, this relationship was no longer significant after adjusting for diagnosis, global functioning, depressive symptoms, deliberate self-harm, mental health outpatient contacts, and type of admission. Weekly hours of care did not significantly impact on length of stay. Patients with carers had poorer functioning which may be related to longer stays. Our analysis was not able to look at subgroups of carers with different needs. Future work is required to determine other components of the admission and discharge process where having a carer is influential.

Keywords: carers; caregivers; hospitalisation; length of stay; mental illness

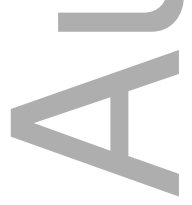

\section{INTRODUCTION}


Informal carers, such as spouses, parents, close friends or even children (Australian Institute of Health and Welfare, 2004) provide intensive ongoing support to people with mental illness; this, in turn, reduces the demands on formal mental health support services. The everyday support provided by mental health carers is varied, and may include emotional support (e.g. encouraging and motivating the care recipient), practical tasks (e.g. managing finances, transportation), and activities of daily living, such as assisting with personal hygiene (Pakenham, 2012, Hielscher et al., 2018). In addition, carers provide support during the acute phases of mental illness (i.e. crisis support), including when the care recipient needs to be hospitalised. Having an informal carer may impact upon several aspects of the hospitalisation process: whether the person gets admitted in the first place (Weich et al., 2014, Giacco et al., 2017), the length of stay and discharge location (Jacobs et al., 2015, Brakoulias et al., 2013), likelihood of post-discharge follow up, and patient outcomes (Reininghaus et al., 2015, Revier et al., 2015). Impact on length of stay is important, as shortening the time spent in hospital may enable other patients in need to access a bed. However, little is known about the impact of carers on length of stay for mental health. Increasing our knowledge on this matter may inform admission and discharge practices, and allow us to gain a better understanding of the impact of mental health carers during the hospitalisation process (Cleary et al., 2006, Semrau et al., 2016, Wood et al., 2013, Giacco et al., 2017).

To our knowledge, only two published studies have specifically explored the impact of having an informal carer on length of hospital stay for mental health (Jacobs et al., 2015, Zhang et al., 2011). In an English study, Jacobs and colleagues (2015) found that seriously mentally ill patients $(n=6,084)$ with an informal carer stayed, on average, three days longer in hospital than those without a carer. This was significant in a pooled model and for patients with schizophrenia or bipolar disorder, but not for patients with schizoaffective disorder. In contrast, Zhang and colleagues (2011) analysed Australian data from an acute adult psychiatric inpatient facility ( $\mathrm{n}=178$ inpatients) and found carer involvement had no influence on length of stay; however, in that study the level of involvement was defined by the treating clinician and not the carer themselves, and therefore carer involvement may have been underrepresented.

Other studies have examined the impact of any family involvement, not just that of informal carers, including whether the patient was brought into hospital by a family member (Brakoulias et al., 2013, Blais et al., 2003, Cyr \& Haley, 1983, Compton et al., 2006) and whether they live with a family member (Wolff et al., 2015, Creed et al., 1997). Most of these studies did not find a significant effect of family involvement on length of stay after adjustment for confounders. The exception was an Australian study (Brakoulias et al., 2013) that reported a significantly longer length of stay for patients brought to hospital by a family member. Jacobs and colleagues (2015) suggested that length of stay may have been prolonged for these patients because their carers required respite from the burden experienced in their caring role. Overall, inconsistent findings in the literature may reflect sampling differences (e.g. study location, type of hospital [public vs. private], diagnoses included), varying definitions of family/carer involvement, as well as differences in statistical methods (e.g. adjustment for confounders (Jacobs et al., 2015) and whether length of stay was continuous or categorical).

Carer burden may also be an important consideration (Jacobs et al., 2015), and could explain more variation in length of hospital stay beyond just presence or absence of a carer. Carer burden is 
typically divided into subjective and objective components; this distinction is important as the incidence and correlates of the two burden types tend to differ (Jones, 1996, Montgomery et al., 1985, Jagannathan et al., 2014). Most studies have focused on subjective burden, i.e. personal appraisal of the caring role including attitudes and emotions (Montgomery et al., 1985, Page et al., 2006, Brouwer et al., 2004). Subjective carer burden has been associated with more psychiatric hospitalisations (Gutierrez-Maldonado et al., 2005, Olawale et al., 2014), and carer stress in particular has been identified as a predictor of longer length of stay for mental health (Draper \& Luscombe, 1998, Tucker et al., 2016). In contrast, objective burden, defined as observable, tangible costs to carers such as monetary costs or disruptions to daily routine (Ampalam et al., 2012, Brouwer et al., 2004, Jones, 1996, Montgomery et al., 1985), has typically been investigated in conjunction with subjective burden (Mansouri et al., 2013, He et al., 2015, Sugiyama et al., 2013). He et al. (2015) found that, after controlling for health insurance, marriage status, diagnosis, and employment status, family burden (both subjective and objective) remained a significant predictor of longer length of stay for patients with mental illness. To our knowledge, no study to date has investigated the impact of objective carer burden alone on length of stay for mental health, using such measures as hours of care per week and standby time or time 'on call' and, in the Australian context, there has only been one investigation of the impact of any form of carer burden on length of stay (Draper \& Luscombe, 1998). Caring for long hours (over lengthy periods of time) tends to be related to caring for someone who is more unwell, coupled with a greater need for carer respite (Jagannathan et al., 2014, Shi et al., 2018), which as hypothesised by Jacobs et al. (2015), could result in a prolonged length of stay for patients. This investigation is therefore pertinent because if objective carer burden is highly associated with longer hospitalisations, attempts to reduce care recipient hospital days may be feasible, for example by targeting more timely and suitable respite options.

\section{AIMS}

The current study was designed to take advantage of the opportunity afforded by two complementary Australian datasets, one covering a national representative sample of people living with psychosis, the other a broader carer community sample. The datasets were complementary as they captured the construct of interest from two different but related and informative perspectives: persons with psychosis were asked directly about whether they had a carer (carer status), as well as mental health hospitalisations in the previous 12 months, whereas the community survey was a broader sample of carers of people with a range of different mental illnesses who were asked questions about objective burden of caring, plus questions about their main care recipient's most recent (mental health) hospitalisation. Therefore, the aims of the current study were to examine:

(1) whether having an informal carer has an impact on length of hospital stay for mental health, and;

(2) whether objective carer burden has an impact on length of hospital stay for mental health.

Considering previous findings (Jacobs et al., 2015, Brakoulias et al., 2013), it was hypothesised that having an informal carer would be associated with a longer hospital stay. Similarly, considering the existing findings on subjective carer burden (Tucker et al., 2016, Draper \& Luscombe, 1998, He et al., 2015), it was hypothesised that higher objective carer burden (i.e. greater hours of care per week) would be associated with a longer length of stay for mental health. The main implications of this study's 
findings will be for adult patients living with a psychotic disorder (for Aim 1), and for carers aged 18+ years who are in contact with carer support organisations (for Aim 2).METHODS

Two Australian surveys were used in this study. The 2010 Survey of High Impact Psychosis (SHIP) was used to address Aim 1, and the UQ Carer Survey 2016 was used to address Aim 2. These analyses were conducted as part of a project on the replacement cost of informal mental health caring in Australia (see Diminic et al. (2017) for further details).

\section{Survey of High Impact Psychosis}

Design. The 2010 SHIP is a nationally representative survey of Australian adults aged 18-64 years with psychotic disorders who were in contact with mental health services. It was funded by the Australian Government Department of Health and Ageing and carried out by the SHIP Study Group (see Acknowledgements) between April 2010 and March 2011. It was conducted in seven mental health catchment areas across five states, with participation from all public mental health services and $86 \%$ of mental health non-government organisations in these areas. The SHIP survey was approved by institutional human research ethics committees at each of the seven study sites and all participants provided written, informed consent. Eligible participants lived in the designated catchment area, were in contact with the above services in March 2010, and screened positive for psychosis. Of 7,955 eligible participants, 4,189 were randomly selected to be contacted for interview and 1,825 completed interviews, a response rate of $44 \%$ (Morgan et al., 2012).

Independent variable (carer status). Participants were asked 'In the last 12 months have you had a carer who is responsible for looking after you, helping you, or taking care of your needs? (Not a paid professional): yes, no, or don't know'.

Dependent variable (length of stay). Participants were asked whether they had been admitted to hospital for a mental health problem in the previous 12 months, and if so the number of times admitted and total nights spent in each of the four facility types (public psychiatric hospital, public psychiatric unit in general hospital, private psychiatric hospital, other). Of participants who were hospitalised in the previous 12 months $(n=626)$, most had one admission $(n=400 ; 64.0 \%), 21.9 \%$ had two admissions $(\mathrm{n}=137)$, and $14.1 \%(\mathrm{n}=88)$ had three or more admissions in the previous 12 months (only $5.6 \%$ had $>3$ admissions)

Analysis of the 2010 SHIP survey focused on whether there was a difference in length of stay for mental health in the previous 12 months for participants with versus without a carer. Participants who had not been admitted in the previous 12 months were excluded from the current analyses, as well as participants with $>1$ hospitalisation over the observation period. The latter was to ensure comparability across the two datasets, as the UQ Carer Survey captured length of stay for the most recent mental health hospitalisation in the past 12 months. Alternative approaches, such as calculating an 'average length of stay' for SHIP participants, would have obscured the data as mental health readmissions within one year tend to be shorter in duration (Heeren et al., 2002). It is worth noting that there was no significant difference in the number of hospital admissions (one versus two or more) in the previous 12 months among participants with versus without a carer $\left(\chi^{2}(1)=2.60, p=.11\right)$. 
Covariates. Based on literature reviews of factors which drive the cost and length of stay in mental health services (Harris et al., 2013, Tulloch et al., 2011), the following covariates and confounders were included in the analysis: sociodemographics (age, sex, country of birth, Indigenous status, primary language), ICD-10 diagnosis (recoded as non-affective psychosis versus other e.g. mania, depression with/without psychosis), course of disorder (recoded as [continuous chronic illness with or without deterioration, or multiple episodes with partial recovery] versus [single or multiple episode with good recovery]), 12-month core depressive symptoms (recoded as presence versus absence of low mood or loss of pleasure), lifetime diagnosis of alcohol or substance abuse/dependence, comorbid serious physical health condition (lifetime diagnosis of diabetes, epilepsy, stroke, heart attack, Parkinson's disease or cancer), global functioning (continuous Personal and Social Performance Scale score), receipt of Disability Support Pension (DSP), deliberate self-harm in the past year, involuntary admissions in the past year (yes/no), private admissions in the past year (yes/no), and number of outpatient contacts for mental health in the past year.

\section{UQ Carer Survey 2016}

Design. The UQ Carer Survey 2016 was an online survey of Australian adults caring for someone aged 16 years or older whose main condition was a mental illness. Ethics approval for survey data collection was obtained from The University of Queensland Behavioural \& Social Sciences Ethical Review Committee (approval number 2015001907). Participants were 105 informal carers of people with a mental illness (schizophrenia, schizoaffective disorder and other psychoses; bipolar disorder; major depression; anxiety disorders; personality disorders; eating disorders; and behavioural disorders) who were recruited from Australian state and territory carer networks and organisations, including members of Mental Health Carers Arafmi Australia (MHCAA) and the Mental Illness Fellowship. All participants provided consent by checking a box below the consent form on the web-based survey. Further details about the survey's methodology can be found in a previous publication (Hielscher et al., 2018).

Independent variable (carer burden). For this analysis, objective carer burden was defined as the average weekly time spent caring for a person with mental illness. In the UQ Carer Survey 2016, carers were asked about the total time spent actively caring for their main care recipient as a result of their mental illness and additional time spent in standby or 'on call'. Participants were firstly asked, "On average, how many hours do you spend each week providing care to your main recipient of care?" Following this they were asked, "Do you spend time in your own home or in your care recipient's home or in close connection to their location so you can be available to them quickly if necessary? If yes, in an average week how many hours do you spend in standby for your main recipient of care?" Hielscher and colleagues (2018) reported on the entire sample $(n=105)$ and found that mental health carers provided on average 37.2 hours of active care per week to their main mental health care recipient. For this analysis, we only focused on the objective burden (i.e. active caring time and standby time) reported by carers who responded 'yes' to the psychiatric hospitalisation question outlined below.

Dependent variable (length of stay). Carers were asked, 'in the past 12 months, has your main recipient of care been admitted to a hospital or residential mental health facility overnight?' If a carer indicated that their care recipient had been hospitalised, they were prompted to answer further questions 
about the most recent hospitalisation including their care recipient's length of stay in days, as well as other questions less relevant to the current analysis such as the type and amount of support postdischarge, including both formal (e.g. psychologist, GP, other health professional) and informal services (i.e. the carer themselves).

\section{Analysis of both surveys}

All analyses were conducted using SPSS version 22.0 (IBM Corp, 2013). For the 2010 SHIP analysis, an independent-samples t-test was conducted to review the unadjusted relationship between carer status and length of stay for mental health. This was followed by linear regression modelling, to examine the effect of carer status on length of stay, controlling for selected covariates/confounders identified from previous reviews (Harris et al., 2013, Tulloch et al., 2011). For each potential covariate, a point-biserial correlation (categorical variables) or Pearson's r correlation (continuous variables) was calculated to assess whether the variable had a significant relationship with length of stay (see Supplementary Material I); variables without a significant relationship were excluded from further analysis. Secondly, point-biserial correlations or phi coefficients were calculated to explore the relationship of each potential covariate with carer status (Supplementary Material I). The relationship between each possible covariate and each other covariate was similarly reviewed (Supplementary Material I). No variables were excluded because each covariate represented a distinct construct, and all correlations between covariates were below a cut-point of 0.3 (Vatcheva et al., 2016). There was also no evidence of multicollinearity based on standard thresholds for the variance inflation factor.

For the UQ Carer Survey 2016, survey data were exported from the SurveyMonkey website into SPSS. In response to average weekly hours of care, two carers reported providing 168 hours of active care to their main care recipient, not including standby time. These outliers were removed prior to data analysis. Analyses were mainly descriptive, involving the calculation of proportions and means. Bivariate correlations using Pearson's correlation coefficient were calculated to investigate the relationship between objective carer burden (i.e. active care time and standby time) and length of stay. A power calculation using $G^{*}$ Power (Faul et al., 2007) found the minimum sample size to achieve $80 \%$ power for detecting correlations of 0.50 or greater, with $95 \%$ confidence, two-tailed, was 29 participants. Bootstrapping was used to calculate $95 \%$ confidence intervals for both proportions and correlation coefficients.

Length of stay transformation. Length of stay (for both SHIP and UQ Carer Survey) was analysed as a log-transformed variable because of the non-normality of the data with extensive skew to the right. This approach has been incorporated in previous investigations of predictors of length of hospital stay (He et al., 2015). Consistent with Jacobs et al.’s (Jacobs et al., 2015) approach, we excluded participants with a very long length of stay, defined as stays over 182 days (approximately 6 months), to reduce the effect of unusually long stay patients on the stability of the estimates and focus on a more homogeneous patient population that reflects the majority of cases seen in the inpatient setting. Six participants were excluded from the SHIP survey (LOS range: 196-329 nights for a single, public hospitalisation); none were excluded from the UQ Carer Survey. Those excluded did not differ in terms of proportion of participants with versus without an informal carer $\left(\chi^{2}=1.27, p=.60\right.$, Fisher's exact test, two- 
tailed), as well as key demographic and clinical characteristics (age, gender, county of birth, Indigenous status, language spoken at home, co-residence status, received disability support pension, number of involuntary admissions, diagnosis, alcohol abuse/dependence).

\section{RESULTS}

\section{Impact of carer status on length of stay (Aim 1)}

The 2010 SHIP survey included 1,825 participants, of whom $620^{1}$ had been admitted to hospital for their mental health in the previous 12 months. Most of these participants ( $\mathrm{n}=394$ or $63.7 \%$ ) had one admission, where they were hospitalised for an average of 29.6 days (Median=21.0, SD=31.08, Range=1-182; logtransformed data $\mathrm{M}=2.87$, Median=3.04, $\mathrm{SD}=1.13$, Range=0-5.20). Of those who had been admitted once, $25.0 \%$ reported receiving help from an informal carer in the previous 12 months. The sociodemographic and clinical characteristics of participants with and without a carer were broadly similar, where the majority were males who were born in Australia, had been diagnosed with nonaffective psychosis, and reported a continuous and chronic course of illness. Patients with carers were significantly more likely to be on the disability support pension, have a lower global functioning score, and were more likely to be chronically unwell, whereas patients without a carer were more likely to have comorbid drug/alcohol abuse or dependence. The full list of variables is listed in Table 1.

[Insert Table 1 about here]

Simple model. In the unadjusted model, length of stay for mental health was significantly higher for participants with a carer $(M=39.18, S D=41.20$; log-transformed data $\mathrm{M}=3.14, \mathrm{SD}=1.15)$ compared to those without a carer $(M=26.52, S D=26.30 ; \log$-transformed data $\mathrm{M}=2.78, \mathrm{SD}=1.11)$, $t(390)=2.74, p=.006, d=0.32,95 \%$ CI of the mean difference [0.10, 0.62]. All t-tests were performed on the log-transformed length of stay variable to adjust for skewness.

Multivariate linear regression. A linear regression model was run to examine whether carer status was associated with length of stay, after adjusting for the effects of diagnosis (affective versus nonaffective psychosis), depressive symptoms, global functioning, deliberate self-harm, mental health outpatient contacts, and involuntary admission. No significant interactions were found so we report on the main effects of variables included the model. The combination of variables were significantly associated with length of stay, $\mathrm{F}(9,315)=5.23, \mathrm{p}<.001$, and explained $13.0 \%$ of the variance (see Table 2 ). Involuntary admission $($ Beta $=.28, \mathrm{p}<.001)$ and self-harm $($ Beta $=-.18, \mathrm{p}<.01)$ contributed significant effects; and together explained $10.2 \%$ of the variance in the model. After controlling for the effects of these other variables, carer status was no longer related to length of stay (Beta $=.09, \mathrm{p}=.12)$.

[Insert Table 2 about here]

Additional analyses. The focus of the main analysis was on 'length of stay'. To achieve comparability with the UQ Carer Survey variable (i.e. length of stay of most recent hospitalisation), we included only SHIP participants reporting a single hospital admission in the previous 12 months.

However, restricting the sample to those with only one admission may have excluded participants that were more unwell/requiring more support, as well as limiting our capacity to do sub-analyses by hospital

\footnotetext{
${ }^{1}$ Total participants hospitalised when the six participants with long length of stays (>182 days) were excluded from the SHIP survey.
} 
type (public versus private). To address these issues, we repeated the above analyses with all hospitalisations in the observation period taken into account, and thus changing the focus from 'length of stay' to one that is more reflective of 'total nights hospitalised', including readmissions/total admissions in the previous 12 months. As seen in Supplementary Material II, total nights hospitalised for mental health was significantly higher for SHIP participants with a carer $(\mathrm{M}=46.88, \mathrm{SD}=52.80$; $\log$ transformed data $\mathrm{M}=3.30, \mathrm{SD}=1.15)$ compared to those without a carer $(\mathrm{M}=37.66, \mathrm{SD}=42.10$; logtransformed data $\mathrm{M}=3.07, \mathrm{SD}=1.16$ ) at the bivariate level. At the multivariate level, the combination of variables were significantly associated with total nights hospitalised, $\mathrm{F}(9,522)=14.62, \mathrm{p}<.001$, and explained $20.1 \%$ of the variance. After controlling for the effects of diagnosis, alcohol abuse/dependence, global functioning, deliberate self-harm, mental health outpatient contacts, involuntary admission, private admission, and number of admissions, carer status was no longer related to total nights hospitalised (Beta $=.02, \mathrm{p}=.56)$. This is consistent with the non-significant carer status results reported in the main analysis. These supplementary analyses also afforded us the opportunity to explore additional variables, such as type of hospital, which was a null finding for public versus private settings.

\section{Objective carer burden and length of stay (Aim 2)}

Table 3 describes the demographic and clinical characteristics of mental health carers (and their main care recipient) who responded 'yes' to the psychiatric hospitalisation question in the UQ Carer Survey 2016. Thirty-eight (or 36.2\%, 95\% CI=26.7-45.7) carers reported that their main care recipient had been admitted to a hospital or residential mental health facility overnight in the previous 12 months. During their most recent hospitalisation, care recipients were hospitalised for an average of 23.6 days (Median= 10.0, $\mathrm{SD}=32.01$, Range= 1-150; log-transformed data Mean=2.45; Median=2.30; $\mathrm{SD}=1.23$ ).

\section{[Insert Table 3 about here]}

In terms of objective burden, carers with a hospitalised care recipient reported providing on average 40.5 hours of care per week to their main care recipient $(\mathrm{SD}=32.5$, Range $=4.5-112.0$; missing data for one participant, two outliers excluded who reported 168 hours of care weekly). In addition, $83.8 \%(95 \% \mathrm{CI}=70.3-94.4)$ reported providing standby time, for an average of 67.9 hours per week $(\mathrm{SD}=63.6$, Range $=1-168)$. Neither average weekly hours of care $(\mathrm{r}=-.20, \mathrm{p}=.31$, post hoc power=.14) nor standby time $(\mathrm{r}=.18, \mathrm{p}=.40$, post hoc power=.37) provided by carers were associated with their care recipient's length of stay in hospital; correlation analyses were performed on the log-transformed length of stay variable to adjust for skewness. Considering both objective carer burden variables were not significantly associated with length of stay at the bivariate level, multivariate analyses were not carried out.

\section{DISCUSSION}

This study contributes to the existing literature by using two complementary Australian datasets, providing different perspectives on the impact of having a carer and length of stay. Results from the 2010 SHIP survey showed that, after adjusting for recipients' diagnosis, global functioning, deliberate selfharm, depressive symptoms, contact with mental health services and type of admission, having a carer did not significantly impact on length of stay. Our analysis of the UQ Carer Survey 2016 found that objective carer burden, as measured by hours of care and standby time, also did not affect length of hospital stay. 
This analysis was the first - to our knowledge - to focus solely on objective carer burden and its relationship to length of hospital stay for mental health.

In terms of the SHIP analysis for carer status, our findings were consistent with the small number of previous similar studies (Jacobs et al., 2015, Zhang et al., 2011, Brakoulias et al., 2013, Blais et al., 2003, Compton et al., 2006, Draper \& Luscombe, 1998, Wolff et al., 2015). Our analysis was particularly robust as it was conducted in a large, nationally representative sample of adults with psychosis; it also investigated length of stay in different types of hospitals (private vs. public; null finding as seen in Supplementary Material II), which has been rarely considered in previous studies. The analysis also adjusted for additional covariates compared with previous studies where carer status significantly impacted on length of stay (Jacobs et al., 2015; Brakoulias et al., 2013). For example, Jacobs et al. (2013) did not control for deliberate self-harm, and neither Jacobs et al,'s (2013) nor Brakoulias et al.'s (2013) study controlled for course of disorder and global functioning. History of self-harm was a significant independent predictor of length of stay in our study, and therefore would have likely impacted on the results of these other studies. Patients with carers were more likely to be on the disability support pension, have a lower global functioning score, and be chronically unwell. Thus, although having a carer did not explain unique variance in length of stay in addition to these variables, it may be that needing a carer and longer hospital stays reflect poorer overall functioning amongst these individuals.

While the current paper considers the presence of a carer as a dichotomous variable, it is possible that carers may not exert a homogeneous effect, and that the average effect presented in the current study may be obscuring systematic differences. For example, a highly stressed carer may benefit from the respite their care recipient's admission provides (and therefore associated with an increased length of hospital stay). Alternatively, a supportive caring environment may give treating teams confidence in an early discharge (and therefore associated with a decrease in length of stay). Carers' stress was not captured in either dataset, preventing us from doing any subgroup analyses, but would be of interest for future research. There are likely other components of the admission and discharge process where having a carer is influential, including whether the person gets admitted in the first place, likelihood of postdischarge follow-up, and patient outcomes. Including carer status in routine admitted patient data collections would allow detailed analysis of carer-length of stay associations using large and nationally representative datasets.

Our analysis of the UQ Carer Survey 2016 found that objective carer burden, as measured by hours of care and standby time, did not significantly impact on length of hospital stay. This is at odds with previous studies on subjective burden (Draper \& Luscombe, 1998, Tucker et al., 2016), as well as subjective and objective burden combined (He et al., 2015) - which show that these variables are significant predictors of length of stay. Subjective carer burden (e.g., carer stress) may be a more useful measure in terms of determining length of hospital stay, compared to objective measures of burden. Subjective burden has been shown to be a stronger predictor (than objective burden) of care recipient distress (Jones, 1996), and other studies have shown that it is not the objective burden that determines level of carer burden, but rather their perception of the caring situation and their ability to cope (MollerLeimkuhler and Madger, 2011). In saying that, the current investigation of objective burden was 
exploratory, and requires investigations in future studies with larger, more representative samples, where the effects of subjective and objective care burden on length of hospital stay can be directly compared.

\section{Limitations}

The UQ Carer Survey 2016 included carers of people with all types of mental illness; however, it was reliant on a small convenience sample which limits the generalisability of its findings. This is particularly relevant to carers who were not in contact with carer support organisations and young carers ( $<18$ years); the latter were excluded from this study to manage ethical implications. When compared to a more representative sample of Australian mental health carers (see Diminic et al. 2017), our sample was similar in terms of marital status, country of birth, language spoken at home, and urbanicity. It is also important to keep in mind that the main implications of this study's findings are for patients with psychotic disorders (SHIP dataset), and primary carers (aged 18+ years) who are in contact with carer support organisations (UQ Carer Survey dataset). Therefore, the current study should be replicated in broader mental health populations, with stratification by key factors (e.g. carer stress). Correlational analyses of the UQ Carer Survey suggest that level of objective carer burden is not associated with length of stay for mental health. However, post-hoc calculations demonstrated that both of these analyses had low power (range $=.14-.37$ ) to detect small correlation coefficients.

Both the 2010 SHIP and the UQ Carer Survey 2016, hospitalisation data were collected via selfreport, and therefore there were likely inaccuracies with respect to recall of nights spent in hospital. The same limitation applies to the hours of care estimates in the UQ Carer Survey 2016Future studies would benefit from collecting diary data on hours of care, which is the gold standard for measuring informal care time (van den Berg \& Spauwen, 2006), as well as hospital admissions data from routine health registers.

\section{CONCLUSION}

This study was undertaken to better understand the role of mental health carers during hospitalisation, particularly their impact on length of hospital stay. The current analysis added to the limited literature on carers and length of hospital stay using Australian data (particularly using nationally representative data), it adjusted for a comprehensive range of key confounders, as well as included a focus on objective care burden, which had not been solely investigated in previous length of stay studies. Consistent with previous studies, our analysis of two Australian datasets found that having a carer, and the level of burden experienced by the carer, did not affect length of stay for mental health, after adjusting for key covariates and confounders in the model. Despite finding no support for the carer status-length of stay relationship, the current study does not discount the importance of building better working relationships between hospital treatment teams and carers, which is viewed by many as one of the key ways forward in mental health care.

\section{RELEVANCE FOR CLINICAL PRACTICE}

People with mental illness who had informal carers in the SHIP survey also had poorer functioning and longer hospital stays, but factors other than having a carer directly predicted length of stay. Deliberate self-harm and involuntary admissions in the past year were highly associated with length of stay, 
consistent with previous studies (Compton et al., 2006; Greenfield et al., 1989), reinforcing the need for treating teams to take account of these factors. Mental health carers in the UQ Carer Survey were providing a substantial amount of support to their care recipient, either in the form of active care hours (average 40.5 hours per week) or time in standby or on call (average 67.9 hours per week). Considering this level of burden, it is important that hospital treatment teams identify and support not just the patient but also their carer during the hospitalisation process and in discharge planning.

\section{References}

Ampalam, P., Gunturu, S. \& Padma, V. 2012. A comparative study of caregiver burden in psychiatric illness and chronic medical illness. Indian Journal of Psychiatry, 54, 239-43.

Australian Institute of Health and Welfare 2004 (28 October 2004). http://www.aihw.gov.au/publicationdetail/?id=6442467651 Accessed: 1 December 2015

Blais, M. A., Matthews, J., Lipkis-Orlando, R., Lechner, E., Jacobo, M., Lincoln, R., Gulliver, C., Herman, J. B. \& Goodman, A. F. 2003. Predicting length of stay on an acute care medical psychiatric inpatient service. Administration and Policy in Mental Health and Mental Health Services, 31, 15-29.

Brakoulias, V., Seymour, J., Lee, J., Sammut, P. \& Starcevic, V. 2013. Predictors of the length of stay in a psychiatric emergency care centre. Australasian Psychiatry, 21, 563-566.

Brouwer, W. B., van Exel, N. J., van de Berg, B., Dinant, H. J., Koopmanschap, M. A. \& van den Bos, G. A. 2004. Burden of caregiving: evidence of objective burden, subjective burden, and quality of life impacts on informal caregivers of patients with rheumatoid arthritis. Arthritis \& Rheumatology, 51, $570-7$

Carers Victoria. 2013 (August 2013). Invisible care: access to Carer Payment and Carer Allowance by Victorian carers of a person with a mental illness. http://www.carersvictoria.org.au/Assets/Files/Carers\%20Victoria_Invisible_Care_Report_2013.pdf Accessed: 1 December 2015.

Cleary, M., Freeman, A. \& Walter, G. 2006. Carer participation in mental health service delivery. International Journal of Mental Health Nursing, 15, 189-94.

Compton, M. T., Craw, J. \& Rudisch, B. E. 2006. Determinants of inpatient psychiatric length of stay in urban county hospital. Psychiatric Quarterly, 77, 173-188.

Creed, F., Tomenson, P., Anthony, P. \& Tramner, M. 1997. Predicting length of stay in psychiatry. Psychological Medicine, 27, 961-966.

Cyr, J. J. \& Haley, G. A. 1983. Use of demographic and clinical characteristics in predicting length of psychiatric hospital stay: A final evaluation. Journal of Consulting and Clinical Psychology, 51, 637-640. 
Department of Social Services 2016 (May 2016). Designing a new integrated carer support service: a draft concept for the delivery of interventions to improve outcomes for carers. www.engage.dss.gov.au/wp-content/uploads/2016/04/Draft-Service-Concept-3.pdf Accessed: 2 June 2016.

Diminic, S., Hielscher, E., Lee, Y. Y., Harris, M., Schess, J., Kealton, J. \& Whiteford, H. 2017 (4 April 2017). The economic value of informal mental health caring in Australia: technical report https://www.mindaustralia.org.au/assets/docs/Mind_value_of_informal_caring full_report.pdf . Accessed: 5 April 2017.

Draper, B. \& Luscombe, G. 1998. Quantification of factors contributing to length of stay in an acute psychogeriatric ward. International Journal of Geriatric Psychiatry, 13, 1-7.

Faul, F., Erdfelder, E., Lang, A. \& Buchner, A. 2007. G*Power 3: A flexible statistical power analysis program for the social, behavioral, and biomedical sciences. Behavior Research Methods, 39, 175191.

Giacco, D., Dirik, A., Kaselionyte, J. \& Priebe, S. 2017. How to make carer involvement in mental health inpatient units happen: a focus group study with patients, carers and clinicians. BMC Psychiatry, 17, 101.

Greenfield, T. K, McNiel, D. E., Binder, R. L. 1989. Violent behavior and length of psychiatric hospitalization. Hospital \& Community Psychiatry, 40, 809-14.

Gutierrez-Maldonado, J., Caqueo-Urizar, A. \& Kavanagh, D. J. 2005. Burden of care and general health in families of patients with schizophrenia. Social Psychiatry \& Psychiatric Epidemiology, 40, 899904.

Harris, M., Legge, N., Diminic, S., Carstensen, G., McKeon, G., Siskind, D., Burgess, P., Stewart, G. \& Whiteford, H. 2013 (30 October 2014). Mental health service cost drivers - an international literature review: final report for Stage B of the Definition and Cost Drivers for Mental Health Services Project, volume 2. https://www.ihpa.gov.au/publications/definitions-and-cost-driversmental-health-services-project Accessed: 25 January 2016.

He, H., Ning, Y., Rosenheck, R., Sun, B., Zhang, J. \& Zhou, Y. 2015. Is severity of family burden a correlate of length of stay? Psychiatry Research, 230, 84-9.

Heeren, O., Dixon, L., Gavirneni, S. \& Regenold, W. T. 2002. The association between decreasing length of stay and readmission rate on a psychogeriartric unit. Psychiatric Services, 53, 76-79.

Hielscher, E., Diminic, S., Kealton, J., Lee, Y. Y., Harris, M. \& Whiteford, H. 2018. Hours of care and caring tasks performed by Australian carers of adults with mental illness: Results from an online survey. Community Mental Health Journal, Online First, 1-17.

IBM Corp 2013. IBM SPSS Statistics for Windows, Version 22.0. Armonk, NY: IBM Corp.

Jacobs, R., Gutacker, N., Mason, A., Goddard, M., Gravelle, H., Kendrick, T. \& Gilbody, S. 2015. Determinants of hospital length of stay for people with serious mental illness in England and implications for payment systems: a regression analysis. BMC Health Services Research, 15, 1-16. 
Jagannathan, A., Thirthalli, J., Hamza, A., Nagendra, H. R. \& Gangadhar, B. N. 2014. Predictors of family caregiver burden in schizophrenia: Study from an in-patient tertiary care hospital in India. Asian J Psychiatr, 8, 94-8.

Jones, S. L. 1996. The Association Between Objective and Subjective Caregiver Burden Archives of Psychiatric Nursing, 2, 77-84.

Mansouri, N., Chimeh, N., Dehghani, M., Malakouti, S. K., Chimeh, N. \& Taherkhani, H. 2013. Risk factors associated with psychiatric hospitalization among Iranian schizophrenic patients. Iranian Journal of Psychiatry and Behavioural Sciences, 7, 16-23.

Montgomery, R. J. V., Gonyea, J. G. \& Hooyman, N. R. 1985. Caregiving and the experience of subjective and objective burden. Family Relations, 34, 19-26.

Morgan, V. A., Waterreus, A., Jablensky, A., Mackinnon, A., McGrath, J. J., Carr, V., Bush, R., Castle, D., Cohen, M., Harvey, C., Galletly, C., Stain, H. J., Neil, A. L., McGorry, P., Hocking, B., Shah, S. \& Saw, S. 2012. People living with psychotic illness in 2010: the second Australian national survey of psychosis. Australian and New Zealand Journal of Psychiatry., 46, 735-52.

Olawale, K. O., Mosaku, K. S., Fatoye, O., Mapayi, B. M. \& Oginni, O. A. 2014. Caregiver burden in families of patients with depression attending Obafemi Awolowo University teaching hospitals complex Ile-Ife Nigeria. General Hospital Psychiatry, 36, 743-7.

Page, A., Hooke, G., O'Brien, N. \& de Felice, N. 2006. Assessment of distress and burden in Australian private psychiatric inpatients. Australasian Psychiatry, 14, 285-290.

Pakenham, K. I. 2012. Caregiving tasks in caring for an adult with mental illness and associations with adjustment outcomes. International Journal of Behavioural Medicine, 19, 186-98.

Reininghaus, U., Dutta, R., Dazzan, P., Doody, G. A., Fearon, P., Lappin, J., Heslin, M., Onyejiaka, A., Donoghue, K., Lomas, B., Kirkbride, J. B., Murray, R. M., Croudace, T., Morgan, C. \& Jones, P. B. 2015. Mortality in schizophrenia and other psychoses: a 10-year follow-up of the SOP first-episode cohort. Schizophrenia Bulletin, 41, 664-73.

Revier, C. J., Reininghaus, U., Dutta, R., Fearon, P., Murray, R. M., Doody, G. A., Croudace, T., Dazzan, P., Heslin, M., Onyejiaka, A., Kravariti, E., Lappin, J., Lomas, B., Kirkbride, J. B., Donoghue, K., Morgan, C. \& Jones, P. B. 2015. Ten-year outcomes of first-episode psychoses in the MRC AESOP10 study. Journal of Nervous and Mental Disease, 203, 379-86.

Semrau, M., Lempp, H., Keynejad, R., Evans-Lacko, S., Mugisha, J., Raja, S., Lamichhane, J., Alem, A., Thornicroft, G. \& Hanlon, C. 2016. Service user and caregiver involvement in mental health system strengthening in low- and middle-income countries: systematic review. BMC Health Services Research, 16, 79.

Shi, J., Chan, K., Ferretti, L. \& McCallion, P. 2018. Caregiving load and respite service use: A comparison between older caregivers and younger caregivers. Journal of Gerontological Social Work, 61, 31-44.

Sugiyama, H., Kazui, H., Shigenobu, K., Masaki, Y., Hatta, N., Yamamoto, D., Wada, T., Nomura, K., Yoshiyama, K., Tabushi, K. \& Takeda, M. 2013. Predictors of prolonged hospital stay for the 
treatment of severe neuropsychiatric symptoms in patients with dementia: a cohort study in multiple hospitals. International Psychogeriatrics, 25, 1365-73.

Tucker, S., Hargreaves, C., Wilberforce, M., Brand, C. \& Challis, D. 2016. What becomes of people admitted to acute old age psychiatry wards? An exploration of factors affecting length of stay, delayed discharge and discharge destination. International Journal of Geriatric Psychiatry.

Tulloch, A. D., Fearon, P. \& David, A. S. 2011. Length of stay of general psychiatric inpatients in the United States: systematic review. Administration and Policy in Mental Health and Mental Health Services, 38, 155-68.

van den Berg, B., Brouwer, W., van Exel, J., Koopmanschap, M., van den Bos, G. A. \& Rutten, F. 2006. Economic valuation of informal care: lessons from the application of the opportunity costs and proxy good methods. Social Science \& Medicine, 62, 835-45.

van den Berg, B. \& Spauwen, P. 2006. Measurement of informal care: an empirical study into the valid measurement of time spent on informal caregiving. Health Economics, 15, 447-60.

Vatcheva, K. P., Lee, M., McCormick, J. B. \& Rahbar, M. H. 2016. Multicollinearity in regression analyses conducted in epidemiologic studies. Epidemiology (Sunnyvale), 6.

Weich, S., McBride, O., Twigg, L., Keown, P., Cyhlarova, E., Crepaz-Keay, D., Parsons, H., Scott, J. \& Bhui, K. 2014. Variation in compulsory psychiatric inpatient admission in England: a crosssectional, multilevel analysis. Health Services and Delivery Research , 2.49.

Wolff, J., McCrone, P., Patel, A., Kaier, K. \& Normann, C. 2015. Predictors of length of stay in psychiatry: Analyses of electronic medical records. BMC Psychiatry, 15, 1-7.

Wood, V. J., Curtis, S. E., Gesler, W., Spencer, I. H., Close, H. J., Mason, J. \& Reilly, J. G. 2013. Creating 'therapeutic landscapes' for mental health carers in inpatient settings: a dynamic perspective on permeability and inclusivity. Social Science \& Medicine, 91, 122-9.

Zhang, J., Harvey, C. \& Andrew, C. 2011. Factors associated with length of stay and the risk of readmission in an acute psychiatric inpatient facility: a retrospective study. Austtralian and New Zealand Journal of Psychiatry., 45, 578-585. 
Table 1. Key sociodemographic and clinical variables of hospitalised participants with and without a carer, 2010 SHIP

\begin{tabular}{|c|c|c|c|c|c|}
\hline \multirow[t]{2}{*}{ Variable } & \multicolumn{2}{|c|}{$\begin{array}{l}\text { Participants with a carer } \\
\qquad(n=98)\end{array}$} & \multicolumn{2}{|c|}{$\begin{array}{l}\text { Participants without a carer } \\
\qquad(\mathrm{n}=\mathbf{2 9 4})\end{array}$} & \multirow[t]{2}{*}{ Bivariate statistic $^{\dagger}$} \\
\hline & $\mathbf{n}$ & $\%(95 \% \mathrm{CI})$ & $\mathbf{n}$ & $\%(95 \% \mathrm{CI})$ & \\
\hline \multicolumn{6}{|l|}{ Gender } \\
\hline Male & 88 & $61.2(51.2-71.7)$ & 185 & $62.9(57.1-68.5)$ & ns \\
\hline Female & 82 & $38.8(28.3-48.8)$ & 109 & $37.1(31.5-42.9)$ & \\
\hline \multicolumn{6}{|l|}{ Country of birth } \\
\hline Australia & 75 & $76.5(67.3-85.0)$ & 235 & $79.9(75.2-84.4)$ & ns \\
\hline Other & 23 & $23.5(15.0-32.7)$ & 59 & $20.1(15.6-24.8)$ & \\
\hline Indigenous status & 5 & $5.1(1.0-9.7)$ & 11 & $3.7(1.7-6.2)$ & ns \\
\hline \multicolumn{6}{|l|}{ Main language } \\
\hline English & 84 & $85.7(78.2-92.7)$ & 264 & $89.8(86.0-93.2)$ & ns \\
\hline Other & 14 & $14.3(7.3-21.8)$ & 30 & $10.2(6.8-14.0)$ & \\
\hline \multicolumn{6}{|l|}{ Mental illness diagnosis (ICD-10) } \\
\hline Non-affective psychosis & 75 & $76.5(67.4-84.7)$ & 197 & $67.0(61.6-72.2)$ & ns \\
\hline Other ${ }^{\ddagger}$ & 23 & $23.5(15.3-32.6)$ & 97 & $33.0(27.8-38.4)$ & \\
\hline \multicolumn{6}{|l|}{ Course of disorder } \\
\hline Continuous chronic illness & 69 & $70.4(61.1-79.0)$ & 178 & $60.5(54.9-65.8)$ & $\mathrm{p}=.048$ \\
\hline Single/multiple episode with good recovery & 29 & $29.6(21.0-38.9)$ & 116 & $39.5(34.2-45.1)$ & \\
\hline Possible depression & 55 & $56.1(46.1-65.9)$ & 186 & $63.3(57.7-68.9)$ & ns \\
\hline Alcohol abuse or dependence & 40 & $40.8(30.8-50.0)$ & 161 & $54.8(48.9-60.6)$ & $\mathrm{p}=.01$ \\
\hline Cannabis abuse or dependence & 75 & $44.1(36.7-51.3)$ & 166 & $56.5(50.8-62.3)$ & $\mathrm{p}=.02$ \\
\hline Other drug abuse or dependence & 45 & $45.9(36.0-55.6)$ & 157 & $35.0(29.5-40.5)$ & ns \\
\hline Physical comorbidity ${ }^{\mathbb{I}}$ & 29 & $29.6(21.0-38.7)$ & 68 & $23.1(18.2-28.0)$ & ns \\
\hline
\end{tabular}

This article is protected by copyright. All rights reserved 
Global functioning ${ }^{\dagger}-\mathrm{M}$ (SD)

Deliberate self-harm in past year

Mental health outpatient contact (M, SD) in past year

${ }^{\dagger}$ Bivariate comparison between SHIP participants with and without a carer on sociodemographic and clinical variables, using Chi-squared or t-tests.

$¥$ Other included mania and depression with or without psychosis.

${ }^{8}$ Core depressive symptoms reported in the previous 12 months.

"Lifetime diagnosis of diabetes, epilepsy, stroke, heart attack, Parkinson's disease or cancer.

${ }^{*}$ Global functioning was measured using continuous Personal and Social Performance Scale score. Higher scores indicate better functioning.

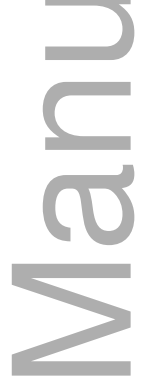

Table 2. Summary of multivariate linear regression of length of stay for mental health, 2010 SHIP (n=394)

\begin{tabular}{|c|c|c|c|c|c|c|}
\hline Variable & B & SE B & $\boldsymbol{\beta}$ & $\mathbf{t}$ & p-value & $95 \%$ CI for $B$ \\
\hline Carer status & .21 & .14 & .09 & 1.58 & .12 & $-.05, .48$ \\
\hline Age in years & .004 & .01 & .04 & 0.74 & .46 & $-.01, .02$ \\
\hline Gender & -.11 & .13 & -.05 & -0.90 & .37 & $-.36, .14$ \\
\hline Psychosis diagnosis & -.03 & .14 & -.01 & -0.19 & .85 & $-.30, .25$ \\
\hline Depression symptoms & .02 & .12 & .01 & 0.16 & .87 & $-.22, .26$ \\
\hline Global functioning & .002 & .004 & .03 & 0.52 & .60 & $-.01,01$ \\
\hline Deliberate self-harm & -.45 & .14 & -.18 & -3.21 & .001 & $-.73,-.18$ \\
\hline
\end{tabular}

This article is protected by copyright. All rights reserved 
Involuntary admission

.63

.12

.28

5.27

$<.001$

$.39, .86$

MH outpatient contacts

.007

.004

1.78

$-.001, .02$

Note. $\mathrm{B}=\mathrm{B}$-weights; $\beta=$ Beta weights; $\mathrm{SE}=$ standard error.

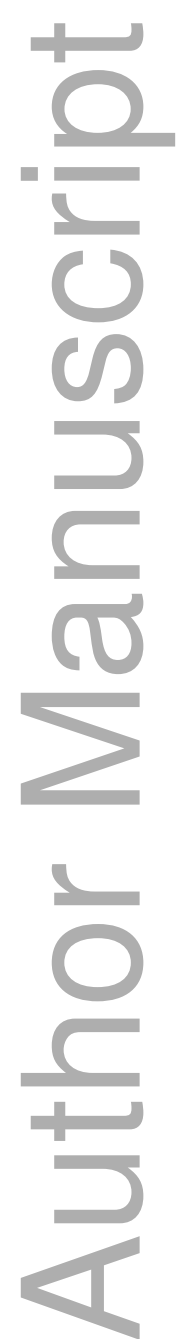

This article is protected by copyright. All rights reserved 
Table 3. Sociodemographics of carers and their hospitalised care recipient in the UQ Carer Survey 2016

\begin{tabular}{|c|c|c|}
\hline Variable & \multicolumn{2}{|c|}{$\begin{array}{c}\text { UQ Carer Survey } 2016 \\
\text { n=38 carer-care recipient dyads }\end{array}$} \\
\hline & $\mathbf{n}$ & $\%(95 \% \mathrm{CI})$ \\
\hline \multicolumn{3}{|l|}{ Age in years } \\
\hline Care recipient - M (SD) & - & $37.87(14.54)$ \\
\hline Carer - M (SD) & - & $55.26(13.38)$ \\
\hline \multicolumn{3}{|l|}{ Gender $\quad \square$} \\
\hline Care recipient - Male & 23 & $60.5(44.4-75.0)$ \\
\hline Care recipient - Female & 15 & $39.5(25.0-55.6)$ \\
\hline Carer - Male & 6 & $15.8(5.3-28.9)$ \\
\hline Carer - Female & 32 & $84.2(71.1-94.7)$ \\
\hline \multicolumn{3}{|l|}{ Care recipient mental illness diagnosis } \\
\hline Schizophrenia or psychosis & 14 & $36.8(21.4-52.6)$ \\
\hline Bipolar, mania & 4 & $10.5(2.4-21.1)$ \\
\hline Depression & 7 & $18.4(5.9-31.4)$ \\
\hline Anxiety & 3 & $7.9(0.0-18.4)$ \\
\hline Borderline personality disorder & 8 & $21.1(8.9-33.3)$ \\
\hline Other & 2 & $5.3(0.0-13.0)$ \\
\hline Care recipient physical comorbidity ${ }^{\dagger}$ & 10 & $26.3(12.8-41.5)$ \\
\hline Care recipient alcohol or drug abuse/dependence & 8 & $21.1(7.9-34.2)$ \\
\hline \multicolumn{3}{|l|}{ Carer relationship: carer is a... } \\
\hline Parent & 22 & $57.9(41.5-74.3)$ \\
\hline Spouse/partner & 13 & $34.2(19.0-50.0)$ \\
\hline Other relative, friend, neighbour & 3 & $7.9(0.0-17.1)$ \\
\hline Lived with carer in last 12 months & 23 & $60.5(43.6-75.8)$ \\
\hline \multicolumn{3}{|l|}{ Length of time being cared for } \\
\hline$>2$ years & 34 & $89.5(78.4-97.6)$ \\
\hline $1-2$ years & 4 & $10.5(2.4-21.6)$ \\
\hline$<1$ year & 0 & 0 \\
\hline \multicolumn{3}{|l|}{ Carer receives government income support ${ }^{\S}$} \\
\hline No payment & 16 & $43.2(26.3-59.4)$ \\
\hline Carer Payment & 6 & $16.2(5.3-29.0)$ \\
\hline Carer Allowance & 13 & $35.1(20.0-50.0)$ \\
\hline
\end{tabular}

Note: in UQ Carer Survey, diagnoses were not verified with a formal diagnostic interview.

${ }^{\dagger}$ Lifetime diagnosis of diabetes, cancer, cardiovascular disease, acquired brain injury, dementia, autism spectrum disorder, intellectual disability, or sensory/speech disorders in UQ Carer Survey.

${ }^{\ddagger}$ These variables were assessed differently in each survey. Alcohol and drug misuse/dependence were included as part of the comorbidity question in UQ Carer Survey, whereas 2010 SHIP survey asked about lifetime diagnoses of alcohol, cannabis, substance abuse/dependence in separate questions. 
${ }^{\S}$ Two points of note. (a) the categories of Carer Payment and Allowance are not mutually exclusive, and (b) some people received other government payments (e.g. age pension, Newstart) which is not shown here.

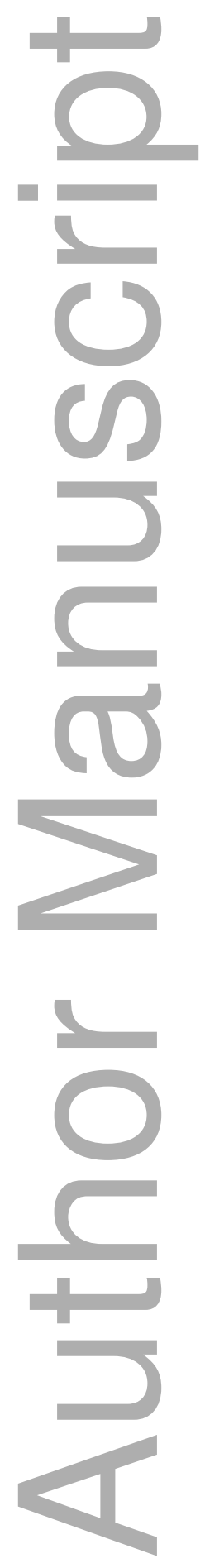

This article is protected by copyright. All rights reserved 


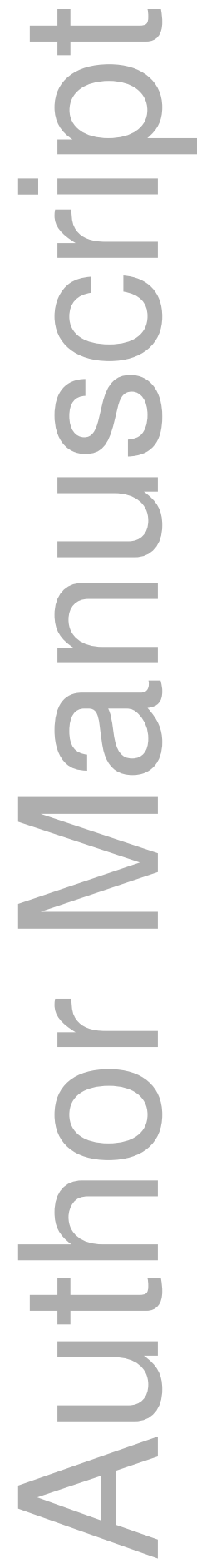

This article is protected by copyright. All rights reserved 


\section{University Library}

\section{- M M N E R VA A gateway to Melbourne's research publications}

Minerva Access is the Institutional Repository of The University of Melbourne

\section{Author/s:}

Hielscher, E;Diminic, S;Harris, M;Castle, D;Lee, YY;Kealton, J;Whiteford, H

Title:

Impact of the carer on length of hospital stay for mental health: Results from two Australian surveys

\section{Date:}

2019-04-01

\section{Citation:}

Hielscher, E., Diminic, S., Harris, M., Castle, D., Lee, Y. Y., Kealton, J. \& Whiteford, H. (2019). Impact of the carer on length of hospital stay for mental health: Results from two Australian surveys. INTERNATIONAL JOURNAL OF MENTAL HEALTH NURSING, 28 (2), pp.436-447. https://doi.org/10.1111/inm. 12543.

Persistent Link:

http://hdl.handle.net/11343/284525 